\title{
Designing A Robot for Elderly Care Homes based on the Notion of 'Robot as Theatre'
}

\author{
Kevin Lefeuvre \\ Bauhaus-Universität Weimar \\ 99423 Weimar, Germany \\ kevin.lefeuvre@uni-weimar.de
}

\author{
Philipp Graf \\ Technical University Chemnitz \\ 09111 Chemnitz, Germany \\ philipp.graf@informatik.tu-chemnitz.de
}

\author{
Eva Hornecker \\ Bauhaus-Universität Weimar \\ 99423 Weimar, Germany \\ eva.hornecker@uni-weimar.de
}

\section{ABSTRACT}

Robots are predominantly thought of as monolithic, unitary actors: they are almost always designed as having a clearly defined body. We rethink this concept of a unified and coherently embodied robot with the example context of elderly care. We explore alternative design spaces for robots in care that open up new modes of interaction for residents and caregivers. We present design studies that explore this notion based on the development of a plant-watering robotic ensemble. The ensemble consists of various elements that inform interacting persons about the robot's task and its role in a storytelling or poetic way. We show how we further explored this idea of a 'robot as theatre' in two different configurations - one agentic and one diorama - and a version that integrates feedback from focus groups with care experts.

\section{Authors Keywords}

Human-Robot Interaction; Elderly care; Agency Attribution; Distributed agency

Permission to make digital or hard copies of part or all of this work for personal or classroom use is granted without fee provided that copies are not made or distributed for profit or commercial advantage and that copies bear this notice an the full citation on the first page. Copyrights for third-party components of this work must be honored. For all other uses, contact the Owner/Author. MUM 2021, December 5-8, 2021, Leuven, Belgium (C) 2021 Copyright is held by the owner/author(s)

ACM ISBN 978-1-4503-8643-2/21/12.

https://doi.org/10.1145/3490632.349787

\section{CSS Concepts}

- Robotics; Interaction design theory, concepts and paradigms; Assistive technologies

\section{INTRODUCTION}

Various technical innovations for the care sector, particularly robots, are pursued to cope with demographic change and support care staff. Analysis of the underlying innovation policy [6] and development practices [7] reveals a gap between innovation discourse and actual fit of such machines for care constellations. Robots for care are normally thought of as autonomously moving entities that interact with elderly (or care staff) or maintain infrastructural tasks. In almost all cases except i.e. the CERO robot [3] - the robotic device is thought of as a unified embodied actor where the whole mechanical device is seen as the robots' body.

The standard design of robots as a single entity conveys that it is one coherent unitary agent. But actually, robots tend to be a conglomerate of components patched together from a number of distributed physical and software entities, some of which may be in the cloud. The perspective of a singular-entity robot is not only empirically false, as such actors are translocally materialised, but it may also not be the best design solution for every robot. We here question this dominant perspective, and envision an alternative and more holistic concept of implementing one or more autonomous entities within the whole device. Thus, the robotic device constitutes an ensemble (or congregation) of interrelated and interactive elements and actors. By doing so, we aim to evoke varying ascriptions of agency to different parts of the robot. Viewers are thereby offered a different interaction script, where they can

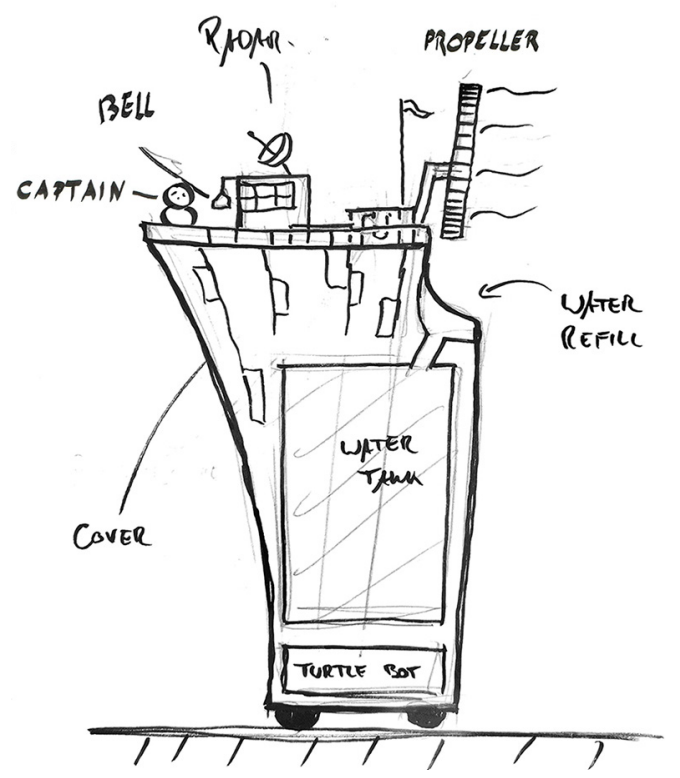

Figure 1: Concept drawing of the agentic concept with one agent and several kinetic elements inhabiting a ship. 
choose between a participating or audience role. The term 'robot as theatre' focuses on one possible outcome: the human steps back and observes the behavior of the robot, fulfilling its task.

An important design implication of every planned robot is the relation between its competences, appearance and task(s) [2]. While competences are needed to fulfill tasks, a robot's appearance should reveal its role and the methods used to do so.

During ethnographic field work in different care homes we identified the issue of boring daily routine and monotony for residents, as well as a lack of indoor plants, as problems that could be addressed in a combined way. Our design exploration investigates how the execution of a utilitarian task - like watering plants could contribute to enriching everyday life. We wanted to develop a poetic or playful device, that appears not as a typical robot, but that entails its own story and is entertaining or pleasant to watch and that may become a topic of discussion. One could argue that the primary function of watering plants becomes a pretext to deeper underlying aims.

By designing a coherent narrative around a device that references well-known role models and stories, we hope to make the object fun to watch within the context of elderly care and offer the role of audience to people. We thus provide choice to those that do not want to interact with the robot or might prefer to simply relate to it in its utilitarian function.

We developed two different approaches based on the 'robot as theatre' notion for the use case of watering plants. This resulted in two detailed concept designs and one prototype. The diorama concept shows a landscape, referring to the flying island Laputa from Studio Ghibli's Castle in the Sky [8], or from Gulliver's universe [12]. We draw on the idea of a scale difference from Gulliver's travels - the small people living on the island deliver water to the plants of the big people, the residents of the care home. From the Studio Ghibli movie we take the idea of a robot that takes care of plants.
The agentic concept references a sea vessel controlled by a small robotic agent, the captain, who takes care of the watering process. The slow-moving ship hosts different elements that the agent interacts with, signaling intentions or machine states to its social environment. For example the captain moderates the watering process by 'using' a control panel. By displaying the agent as being in control of the ship's movement and actions, we aim to elicit the illusion of an interactive process that is interesting to watch.

In this contribution, we focus on the underlying approach and metaphor of the 'robot as theatre' and two variations of this alternative HRI conception, based on the example of a plant-watering robot (PWR). So far, only one of the designs has been previously introduced briefly, with a focus on evaluating its mode of signaling in a videobased study [9]. We here add detail on the underlying notion of 'robot of theatre' and, by presenting both approaches, allow a comparison.

We further report feedback and design suggestions from focus group sessions based on a video prototype on the PWR and present an alternative agentic design. This design version integrates the new suggested use case of a mobile and more interactive record player device, the mobile Jukebox.

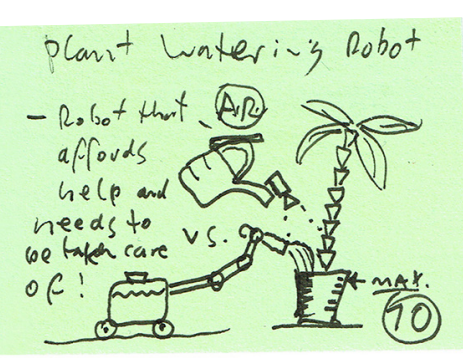

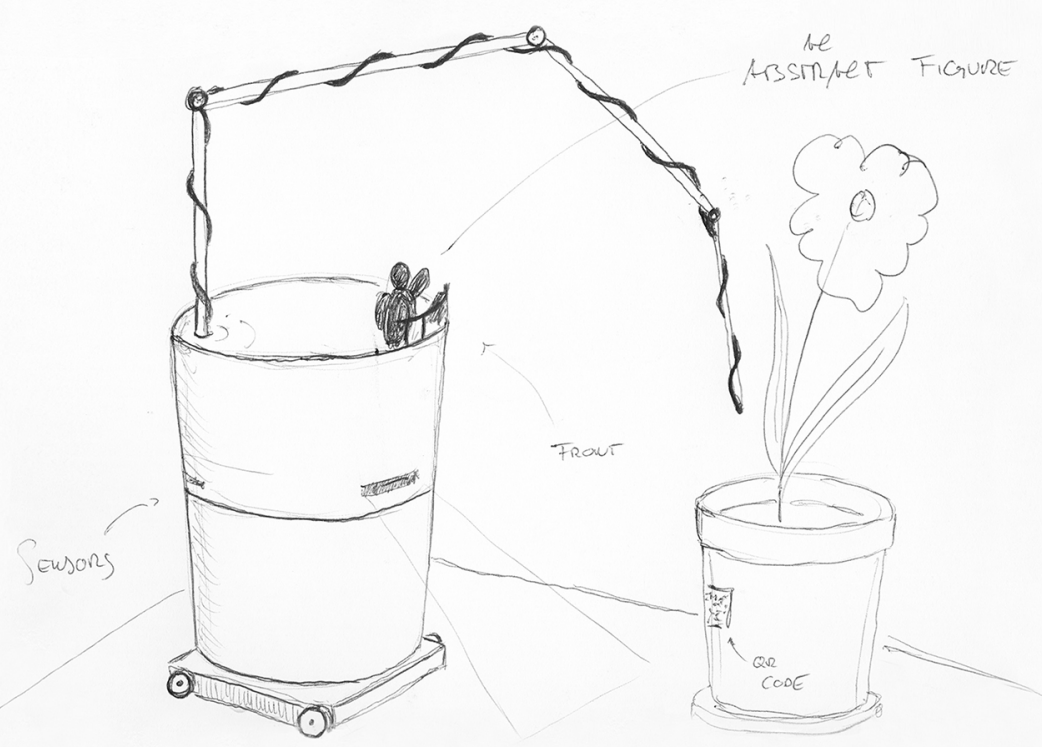

Figure 2 (top right): The post-it sketch depicts another variation of an assistive device that can be attached to a conventional watering can to help residents care for plants.

Figure 3 (bottom): Preliminary sketch of a plant watering robot that autonomously takes care of plants. 


\section{ROBOT AS THEATRE}

The core idea is to consider the robot not as a unified agent, but as an embodied ensemble of various elements with different functions and levels of agency, that together constitute the robotic functionality. All these elements sit on a platform, which in our case can move. The robot should not require interaction from humans but tell an interactive story in (and on) itself. In early phases of the design process, we explored various options for providing the impression of an 'inhabited robot'. Several versions for a main figure representing the direction of movement and watering for the robot were developed, in search of a suitable metaphor and character that people in an elderly care home would consider likeable and that would convey the functionality of our robot.

This led to the metaphor of the 'robot as theatre', where the device as a whole serves as a stage, on which the interaction between various agents and props tell a story about the robot's purpose and actions. In the next step, we decided to put more focus on the stage itself and to design the robot as a mini-world for the specific context of elderly care. Having a mini-world inhabited by different actors and interacting objects could make the robot entertaining to watch, allows for rich storytelling, and would be true to the metaphor of theatre, where the stage design contributes to the meaning of a play. Depending on the concrete concept, diorama or agentic version, different entities and control relations are displayed. We thereby hope to elicit different agency ascriptions within one device.

Our conceptual robot design builds on the metaphor of 'computers as theatre' introduced by Brenda Laurell [5], who described the use of computers and interfaces as something that is symbolic and representative, with an observer that places themself either in the audience or even within the play, thereby sharpening our understanding of such interactions. Yet since our concept is not intended as an interface for users, but as a world in its own right, we modified this metaphor to a 'robot as (mobile) theatre' underlining its mobile and playful character.

The robot is supposed to intervene in the living space of the residents and to interact with elements of this space, the plants. There thus is no clear separation between stage and audience. This non-strict separation, which may even include interaction with the audience, is reminiscent of the old Shakespearean theatre tradition.

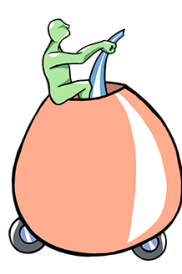

Attempt to depict an old water jug.

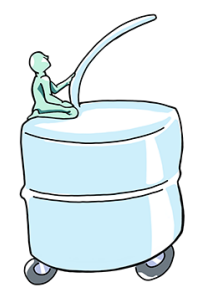

Sitting person (more passive)

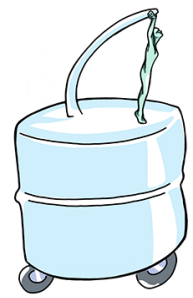

Standing person (more active).
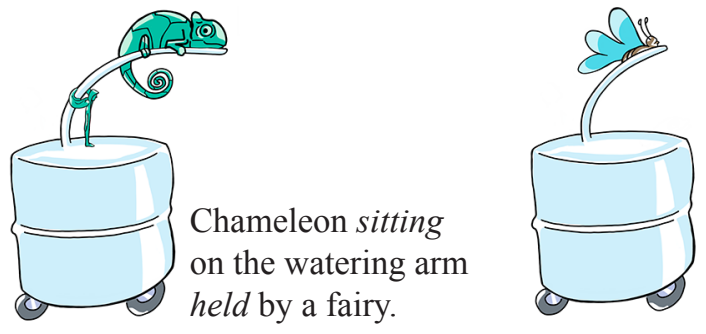

Butterfly sitting on the watering arm.

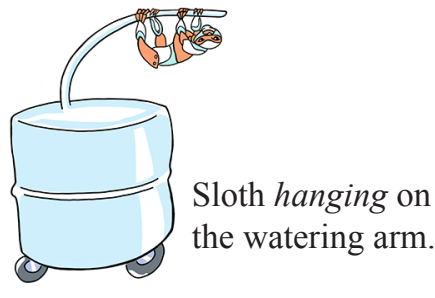

Figure 4: Sketches exploring options for character design for the agent(s) of the PWR. Besides of a human figurine (which might raise expectations about being able to talk with it), we considered figures of animals related to plants that would convey a sense of tranquillity and be gentle and cute.

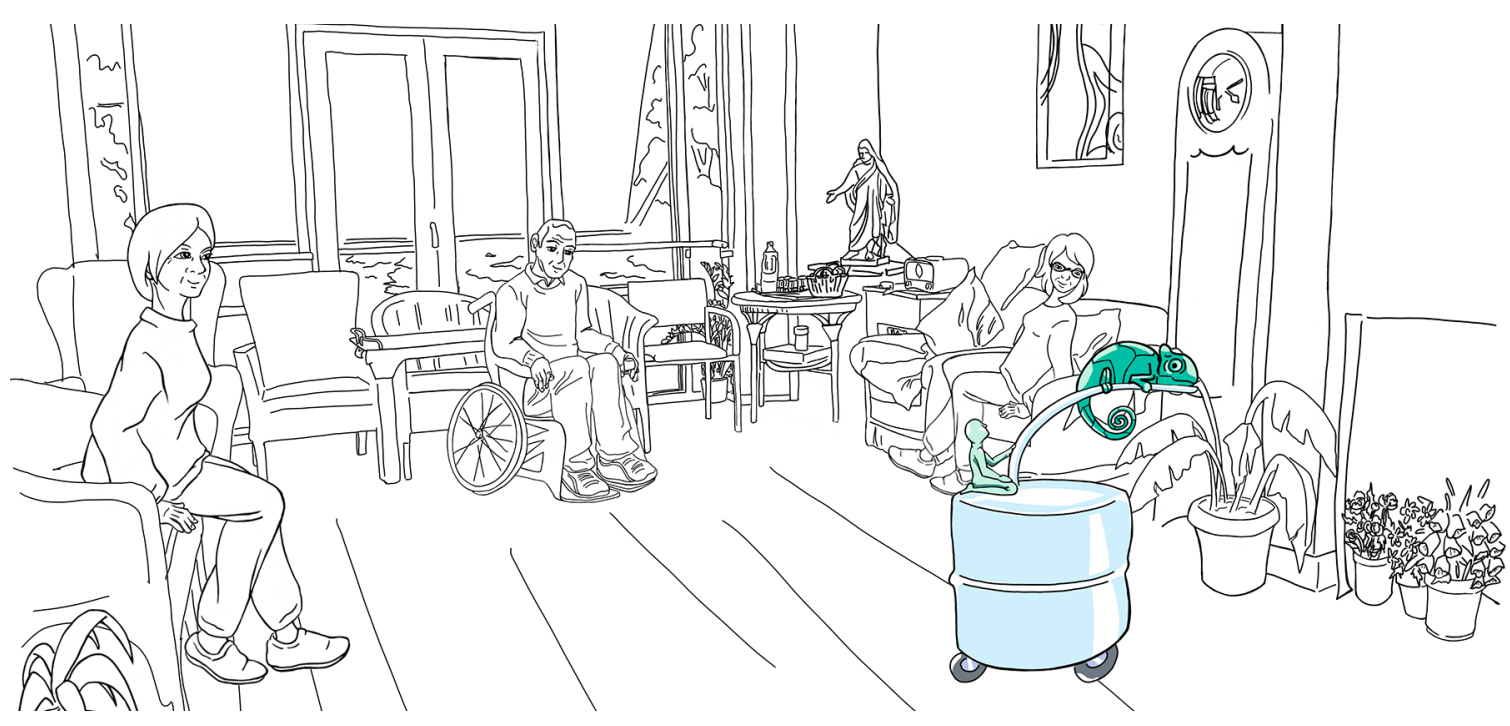

Figure 5: Sketch of the PWR in elderly care home. 

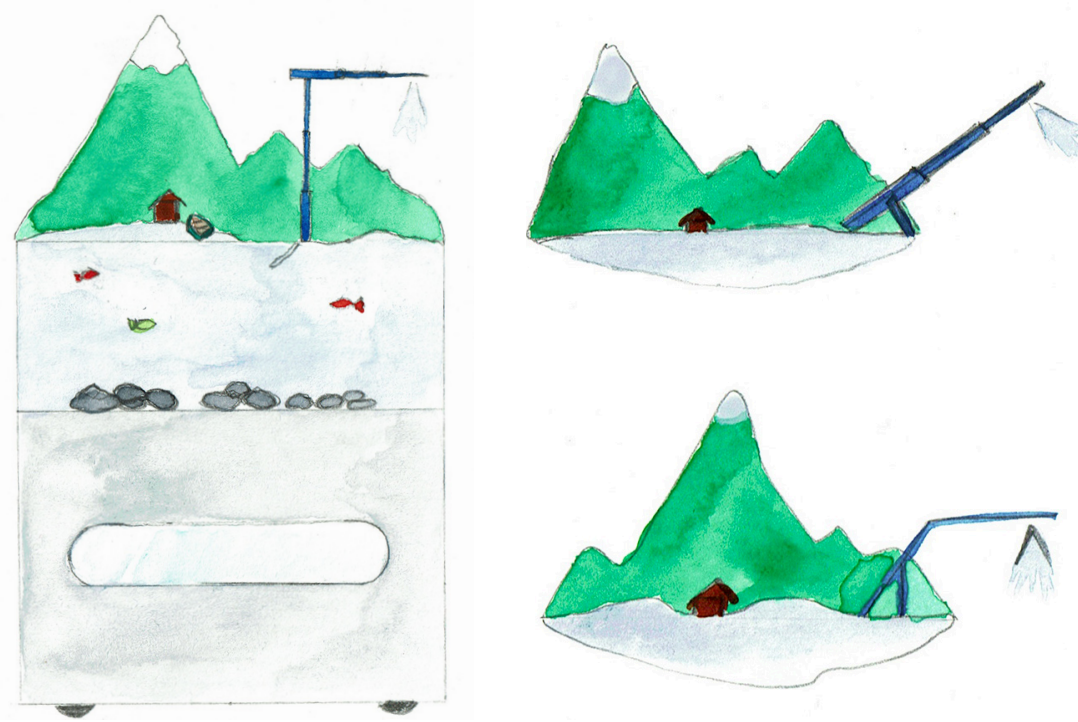

Figure 6: Sketches for the diorama concept with variations of the watering arm.

\section{TWO APPROACHES FOR 'ROBOT AS THEATRE': DIORAMA AND AGENTIC}

Two concepts were designed in detail. We then assessed which appeared more appropriate for our specific application context for further development and prototyping. Note that while we selected one approach, the other could be suitable for other applications, tasks or use contexts. For both approaches, the basic requirements (or design constraints) were similar. Because of the watering function, water should be an indispensable component of the overall design concept. The relation between water, the displayed world of the robot, and its task has to be legible for an audience. Thus, finding a narrative around water was the starting point for developing the two concepts.

Since the robot autonomously executes its utilitarian but pretext function of watering plants, and only needs to be observed for its entertainment function, its design should not suggest that any direct interaction is needed. Moreover, the robot should be introduced as a functional, utilitarian object, so that the audience does not feel obliged to be amused, and is free to ignore the robot. Both the concept of a closed miniature world and that of a robotic ship hosting an agent and other interacting elements meet these criteria. While the diorama version entails a more poetic and abstract storyline, the ship, on the other hand, is centred on a main actor, the ship's captain, who is accompanied by several kinetic elements, which give the ensemble a utilitarian and concrete narrative.
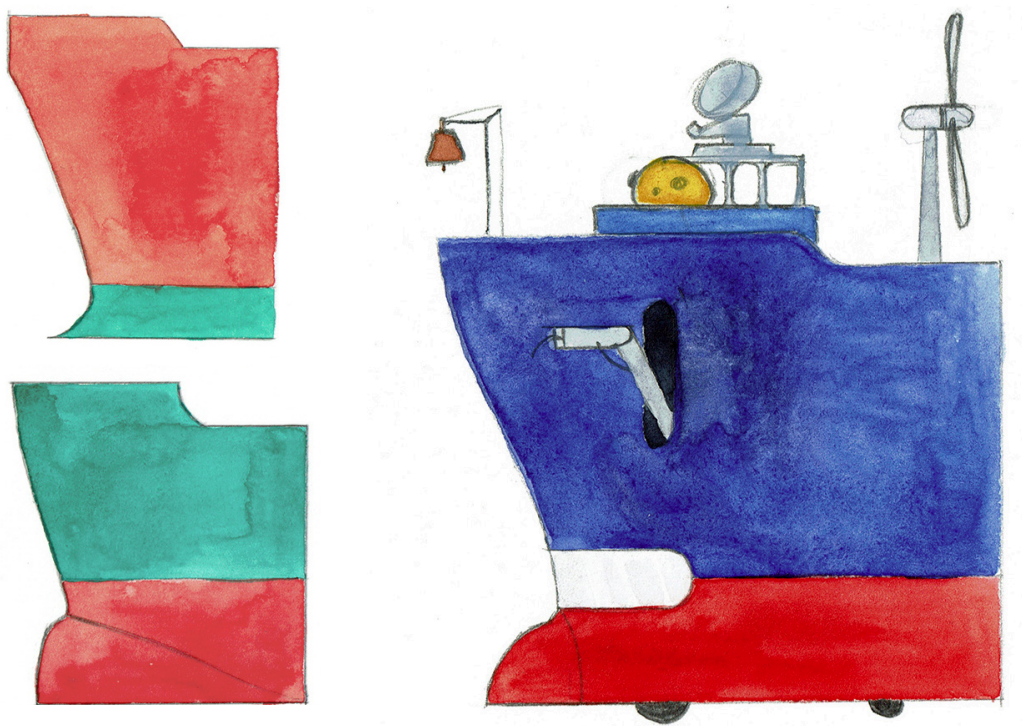

Figure 7: Sketches for the agentic concept with variations of the ship hull.

Another cultural reference for our concepts - besides theatre - is the world of automata, complicated mechanical devices that resemble living beings and sceneries built in the last centuries - peaking in the 18th century. Automata simulate capabilities of humans or animals - often including context with objects - or interactions between different entities within a mechanical device [15]. Although automata were often planned or built with an epistemic purpose [11], their practical function often was to be shown to an admiring audience.

We also refer to the concept of dioramas, 3D scale models of landscape, often behind glass or a barrier $[10,13,16]$, showing historical, imagined or nature scenes. In museum dioramas, original exhibits are integrated into a small landscape creating context. Dioramas are there to be viewed and inspected, but do not invite interaction. Frequently, they use static or moving elements of storytelling. In museums, they provide entertainment, enjoyment, and stimulation, and give course for conversations Such miniature worlds may also be built by hobbyists as part of model railway scenery or for board games miniature figure modeling.

In the following we describe the diorama and agentic approach of the 'robot as theatre' in more detail, focussing on design choices and their rationale. 


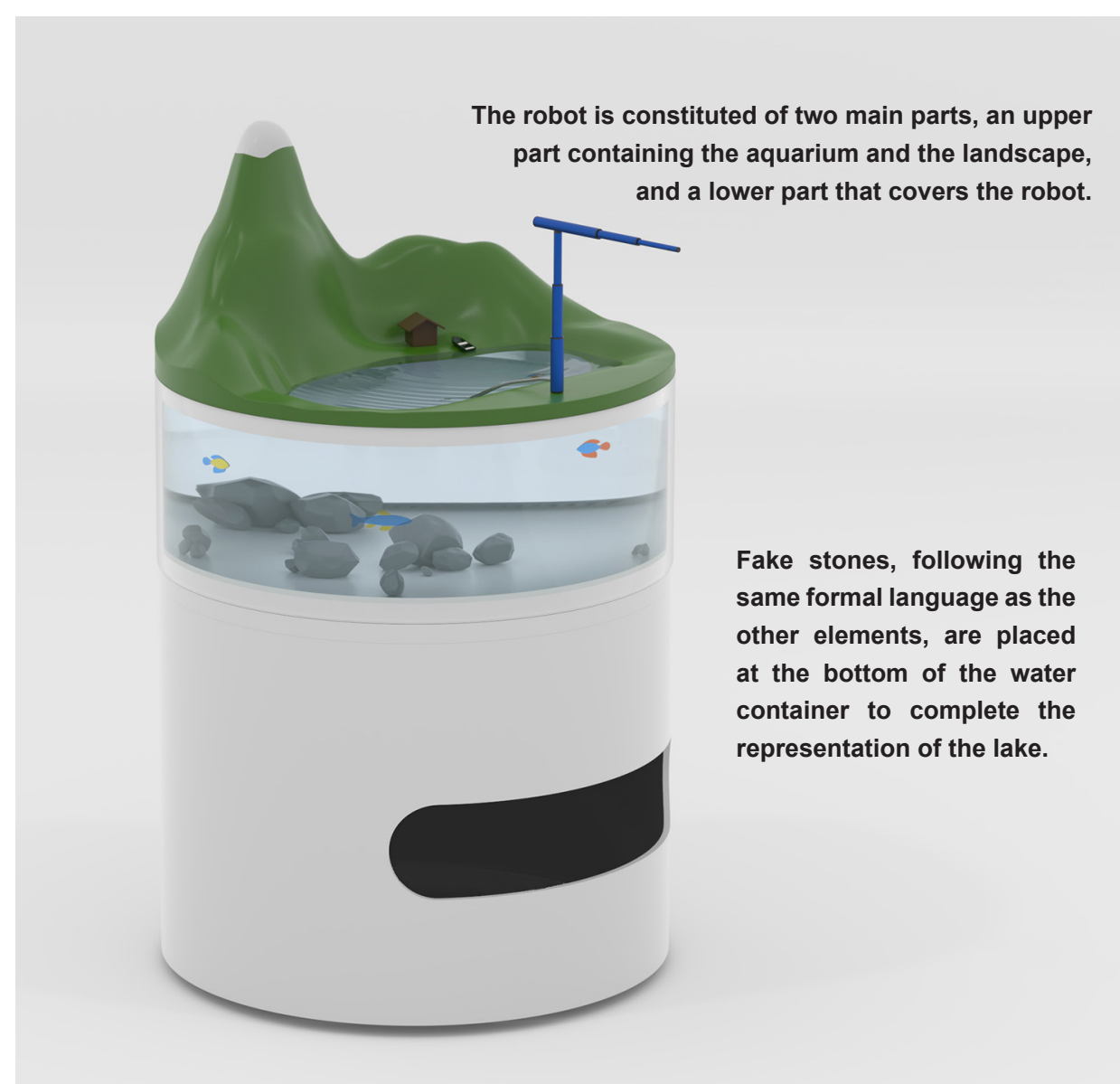

Figures 8, 9 \& 10: Computer Generated Images (CGI) of the diorama concept.

\section{DIORAMA CONCEPT}

Our concrete diorama concept is inspired by the islands of Laputa and Lilliput from Gulliver's Travels [12]. Like the former, it is a moving, mobile world, and its scale resembles that of the latter. The overall shape of the diorama is a circle, the abstract shape of an island. Since water is essential for the robot, the diorama represents a mountainous landscape with a lake as the central element. The interaction between this moving island and the outside world is symbolised and made functional by a giant (regarding the scale of the island) watering machine that draws water from the lake. The inhabitants of this moving island have the task of watering the plants of our human
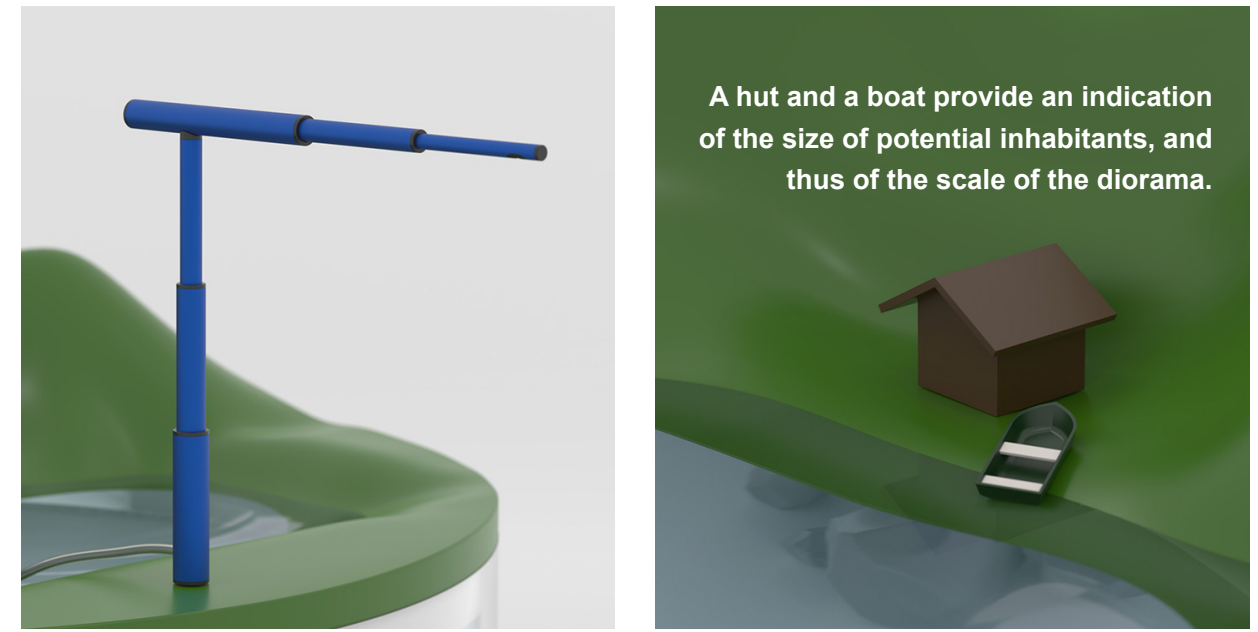

world. The underside of the lake is visible through a transparent wall and is used as a mobile aquarium that holds (robotic) fish.

To make this robot pleasant and entertaining to look at, it is not enough to integrate moving elements such as the watering machine and fish. The shape, materiality and the $3 \mathrm{D}$ quality of the diorama also plays an important role. The product language, made of primitive forms and uniform colours, is intended to be abstract, so that it can be easily understood without being childish. Also, a very slow movement behavior should resemble a big and ponderous moving island - this would also be necessary in order to keep the water level stable.

Although this version did not get realized as a physical prototype, the design did consider how the robotic technology could be integrated. The bottom part of the robot consists of a cover, hiding a TurtleBot3 Waffle Pi [14], a robot platform available on the market, in charge of mobility and the watering process. The water pump is located between the landscape and the water container, and connected electronically to the TurtleBot via a junction element at the robot's rear. Because the TurtleBot3 Waffle Pi utilizes an optical sensor to navigate, an opening is required at the bottom of the covering.

As this concept is not only scaled differently to the outer world - the care home - but also regarding its inner world - a giant water pump and possibly giant agents - an implementation seemed to be rather complicated. In particular, a well synchronized behavior of these elements that would appear 'natural' despite these scale differences, would be difficult to realize. Moreover, we anticipated that elderly residents would be surprised by a moving landscape, the metaphor hard to understand for everyone. 


\section{AGENTIC CONCEPT}

Our second concept also represents a small-scale world that interacts with our world. Different to the landscape concept, the design choice of a vehicle enables a more coherent product regarding its scale and interrelating objects, with only one central actor. Since it depicts a vehicle, we hope the reference is better known and thus its mobility should be less surprising for residents of an elderly care home.

As water is the central element, we designed this as a small deep sea vessel. The ship's deck, which contains various kinetic elements, is the most interactive part and constitutes the 'stage' of the robot. The additional archetypical elements exploit the metaphor of a ship and its captain as props with which the story of the small captain controlling the vessel is being told. Instead of having one unified body, this robotic device appears as consisting of at least two different entities, the agent and its ship plus individual additional elements. The kinetic character has the role of the ship's captain, who controls the ship. To realize this, we utilized a small robot available on the market, a My Keepon [1, 4], a small robot with an abstract shape that can turn on itself and bounce its body.

Each kinetic element has an informative function, from a ringing bell, a rotating radar for showing search direction, a propeller indicating direction of movement, to blinking LEDs for the watering status. The watering process is carried out by a water-hose arm on the left side of the ship. With this design, we hope to foster the robot's entertaining potential, while at the same time providing viewers with an understanding of what the robot does or is planning to do next, thereby enabling the residents to anticipate its behavior.

An opening at the rear of the ship's deck enables it to check the water level and fill the tank. Like the diorama concept, an opening in the bottom of the ship is necessary for the optical sensor used for the autonomous navigation.
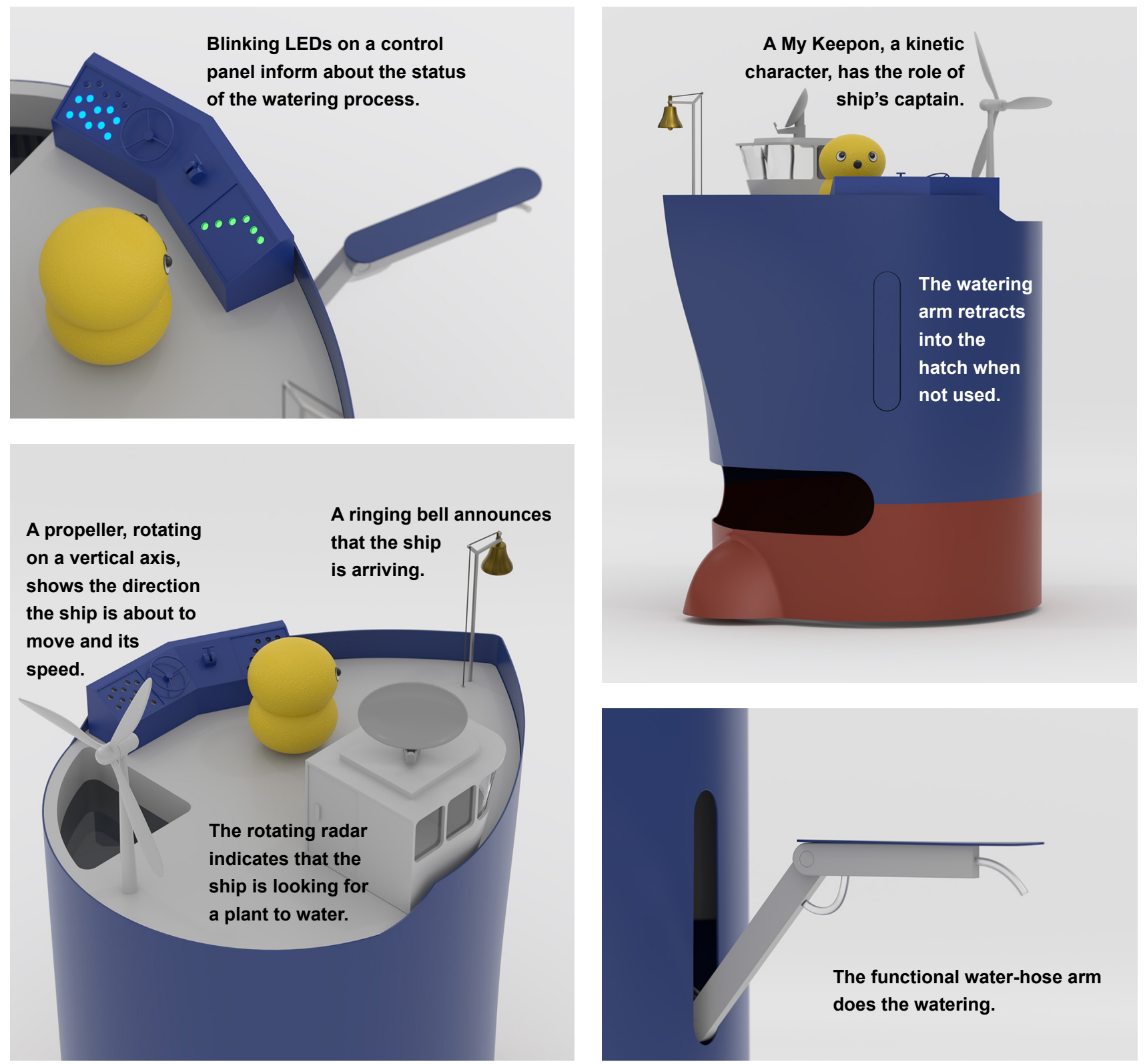

Figures 11, 12, 13 \& 14: CGIs of the agentic concept. 

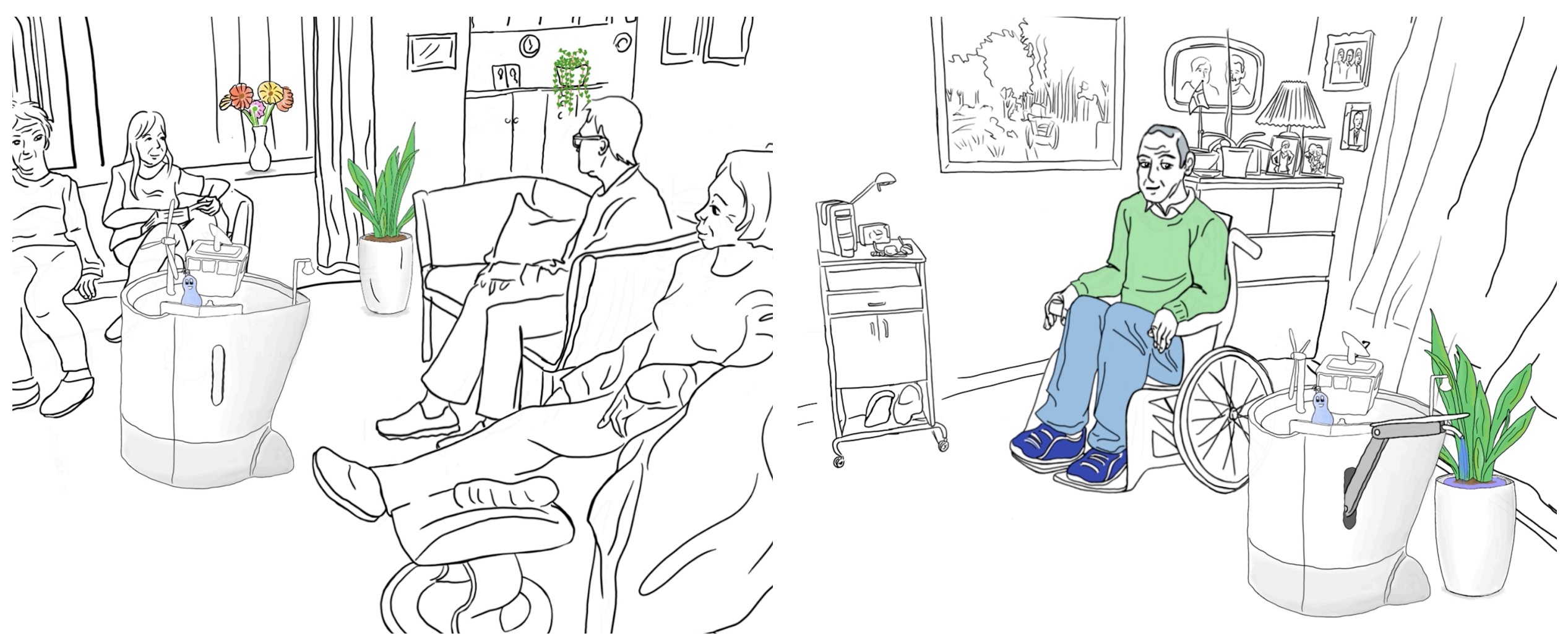

Figure 15: Pictures extracted from the video prototype, showing the ship approching a plant and watering it. On the left in a common room, and on the right in a private room.

\section{FOCUS GROUPS FEEDBACK}

The functionality and design of the agentic version of the PWR was discussed in focus groups with professional and family caregivers. We developed a video prototype that displays the ship, titled M.S. Ilma, driving around an elderly care home. The video shows the double function of M.S. Ilma focussing on its storytelling aspects and appearance. While the overall concept of a double function as well as the notion of a mobile theatre was discussed positively, the concrete function of watering plants was found to be of minor relevance. Although none of the participants suggested that the risk of deception was too high, the consensus was that empirical tests need to ensure the design is not perceived as childish.

Other possible 'pretext' tasks were suggested for the agentic concept, such as reading analog media to residents, broadcasting news, playing music (Jukebox) or serving as a sort of everyday helper or companion robot for residents. We decided to further develop the idea of a mobile jukebox, as it builds strongly on an existing and fairly known device (at least for older generations). Similar to the PWR concept it should be controlled by an agent, but in this case one that interacts more directly with residents. 
The My Keepon next to the turntable appears to operate an arm to change records.

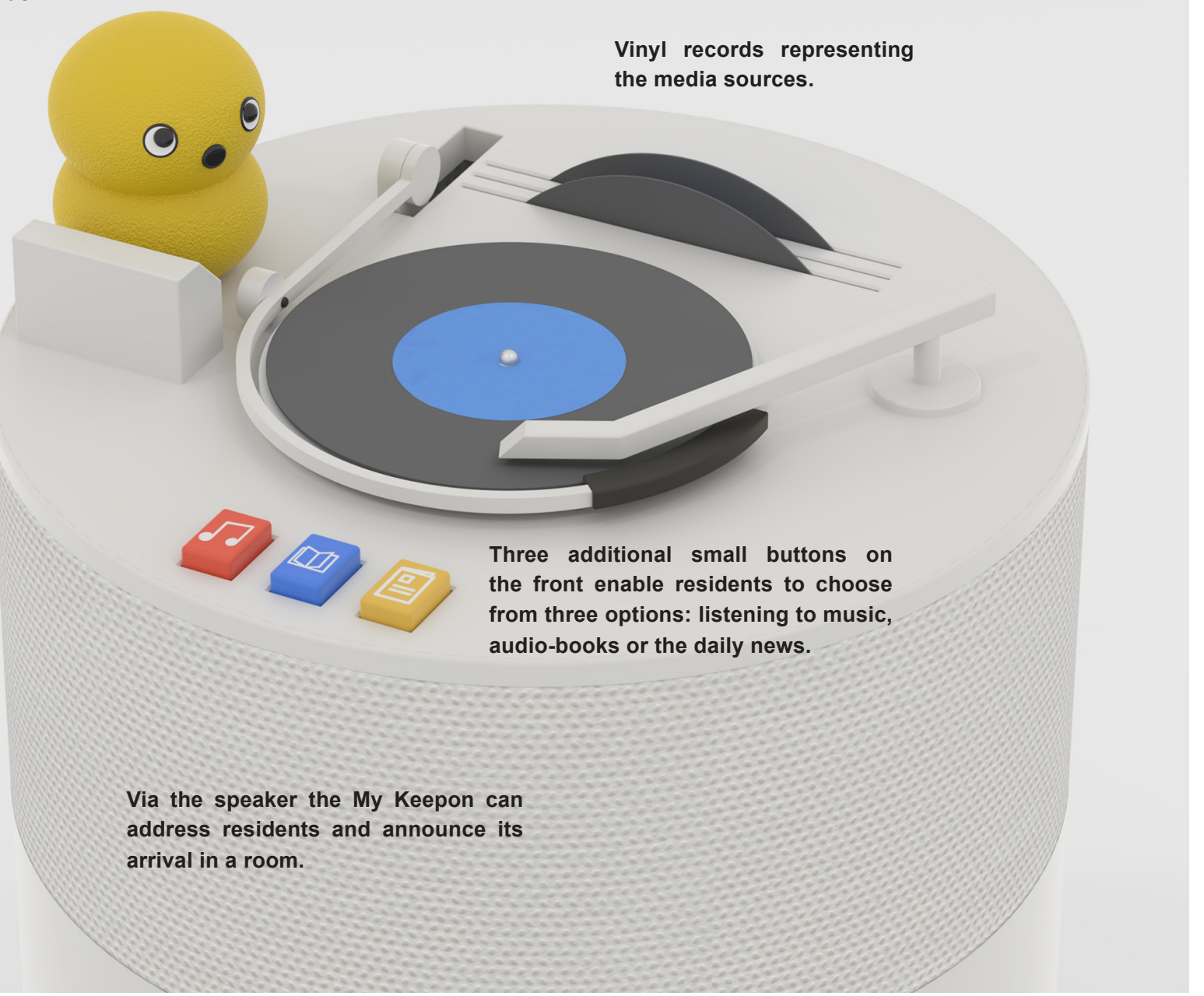

\section{JUKEBOX}

We combined several suggestions of the focus groups and combined them into the mobile jukebox concept a mobile speaker with an agent that is operated via voice recognition, which would broadcast news, play records or sing with residents. In contrast to the other two concepts, the agent engages and invites residents to interact more directly with the robot, instead of just being spectators. Just as the diorama concept, the jukebox concept is constituted of two sections, the robot base and an upper section, on which a turntable and a small supply of vinyl records are displayed, operated by a My Keepon. A possible addition could be a transparen cover similar to those of traditional record players to protect from dust or manual interferences.

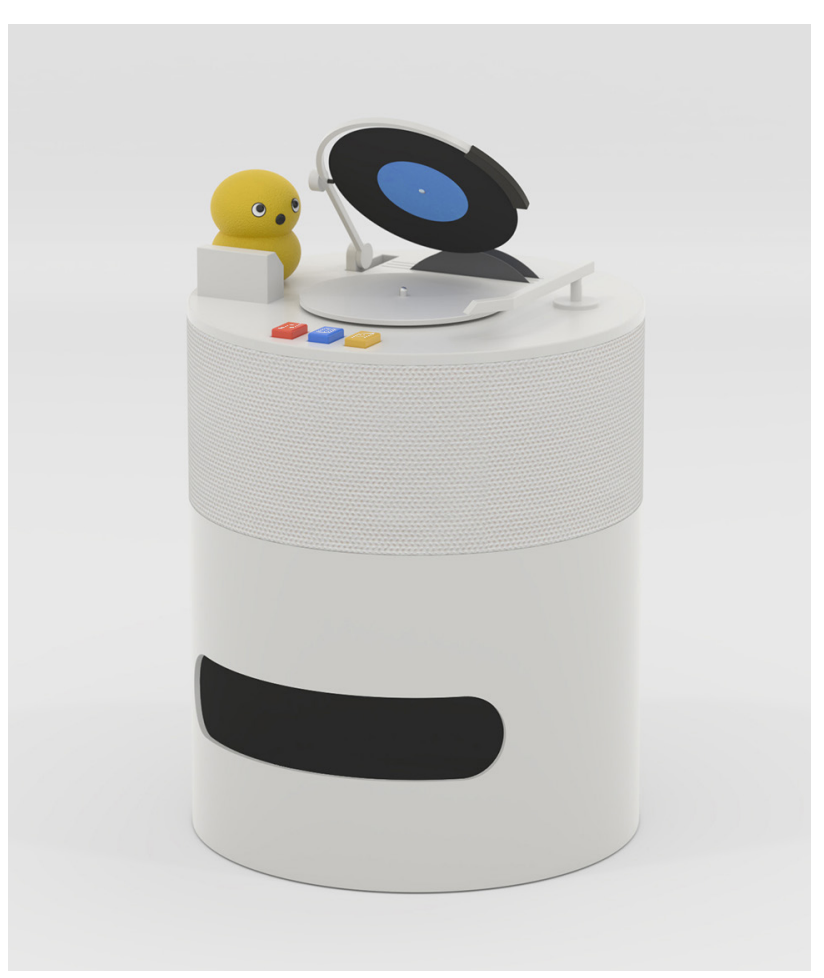

Figures 16: CGIs of the jukebox concept. 


\section{PROTOTYPE AND FUTURE RESEARCH}

We selected the agentic design version for prototyping development, because we believe that a small agent interacting with a vehicle will be intuitively more understandable to residents. The My Keepon, which easily elicits feelings of sympathy, and the diversity of interactive, multimodal elements, which can support storytelling and understanding for an observer, are further advantages over the diorama concept in the context of elderly care homes.

A first prototype with a 3D-printed shell was built to test elementary functions, such as the orientation signalling by the My Keepon and the propeller [9]. The shell of the prototype was printed on a smaller scale due to manufacturing constraints. A second prototype in full scale with all elements will be built for empirical testing in an elderly care home.

\section{CONCLUSION}

We contribute an exploration of alternative design concepts for robots, rethinking the dominant conception of robots as monolithic, unified entities. In this pictorial, we explored a design approach of robots as a world in its own right and scale, portrayed either as a congregation of agents in an ensemble or as an abstract miniature landscape (diorama) with invisible inhabitants.

The core of our contribution is an exploration of the metaphor of the 'robot as theatre', where there is one moving platform which provides the stage for various potentially agentic elements, while at the same time implying that human viewers can take the role of audience. This supports the intended role of our robot, to fulfill a utilitarian task of watering plants in a care home, while providing entertainment and a talking point, without requesting direct interaction. We further investigated the notion of 'robot as theatre' through two design concepts, the 'diorama' and 'agentic ensemble'. Based on feedback from care workers, we then developed the third concept of the mobile Jukebox. With these three alternative robot designs, we illustrate an emerging design space for non-unified robotic devices that are intentionally designed as conveying distributed agency, while at the same time providing a playful and entertaining performance for viewers.

We suggest that this design approach may be more appropriate for representing the fact that robotic functionality is not the result of one entity, but often actually consists of several inter-operating components, where there is not one locus of control, but a distributed system. This approach may on the one hand support an intuitive understanding of distributed agency in robots, and on the other hand provide an option to adequately represent the often fragmented agency of robotic actors.

\section{ACKNOWLEDGMENTS}

This research was supported by the project ReThiCare, funded by the VolkswagenStiftung.

We thank Emanuela Marchetti for the sketches in figures 4, 5 and 15. For further contribution to figure 15 and video prototype we thank Mira Thieme. We also thank our project partners at SDU Odense, Norbert Krüger, Lakshadeep Naik, Christian Sønderskov Zarp and Oskar Palinko, for the technical development based on our initial concept and for discussion. We thank the reviewers for their valuable feedback.

\section{REFERENCES}

[1] BeatBots. 2021. My Keepon. Retrieved September 19, 2021 from https://beatbots.net/my-keepon

[2] Jennifer Goetz, Sara Kiesler, and Aaron Powers. 2003. Matching robot appearance and behavior to tasks to improve human-robot cooperation. In Proceedings of the 12th IEEE International Workshop on Robot and Human Interactive Communication (ROMAN 2003). January 2003, 55-60. DOI:https:// doi.org/10.1109/ROMAN.2003.1251796

[3] Helge Hüttenrauch and Mikael Norman. 2001. Pocket CERO - mobile interfaces for service robots. In Proceedings of Mobile HCI 2001: Third International Workshop on Human Computer Interaction with Mobile Devices (IHM-HCI 2001), September 2001, Lille, France, 1-7.
[4] Hideki Kozima, Marek P. Michalowski, and Cocoro Nakagawa. 2009. Keepon: A Playful Robot for Research, Therapy, and Entertainment. Int J of Soc Robotics 1, 1 (January 2009), 3-18. DOI:https://doi org/10.1007/s12369-008-0009-8

[5] Brenda Laurel. 1993. Computers as Theatre (2nd. ed.). Addison-Wesley Longman Publishing Co., Inc., USA.

[6] Benjamin M. Lipp. 2019. Interfacing RobotCare. On the Techno-Politics of Innovation. Doctoral thesis. Technical University of Munich. Munich.

[7] Arne Maibaum, Andreas Bischof, Jannis Hergesell, and Benjamin Lipp. 2021. A critique of robotics in health care. $A I \&$ Soc (April 2021). 1-11. DOI:https://doi.org/10.1007/s00146-021-01206-Z

[8] Hayao Miyazaki, James Van Der Beek, Anna Paquin, and Cloris Leachman. 2012. Castle in the sky. [United States], Disney.

[9] Oskar Palinko, Philipp Graf, Lakshadeep Naik, Kevin Lefeuvre, Christian S. Zarp, and Norbert Krüger. 2021. Intention Signaling for Mobile Social Service Robots - The Example of Plant Watering. In: Li H. et al. (Ed.) Social Robotics. ICSR 2021. Lecture Notes in Computer Science, Vol. 13086. Springer International Publishing, Cham, 797-802. DOI:https://doi.org/10.1007/978-3-030-90525-5_74

[10] Michael J. Reiss and Sue Dale Tunnicliffe. 2011. Dioramas as Depictions of Reality and Opportunities for Learning in Biology: Dioramas as Depictions of Reality and Opportunities for Learning. Curator: The Museum Journal 54, 4 (October 2011), 447-459. DOI:https://doi.org/10.1111/j.21516952.2011.00109.x

[11] Jessica Riskin. 2003. Eighteenth-Century Wetware. Representations 83, 1 (August 2003), 97-125. DOI:https://doi.org/10.1525/rep.2003.83.1.97 
[12] Jonathan Swift. 1667-1745. Gulliver's Travels into Several Remote Regions of the World. Retrieved November 4, 2021 from http://www.gutenberg.org/ ebooks/17157

[13] Sue Dale Tunnicliffe and Annette Scheersoi (Ed.). 2015. Natural History Dioramas. History, Construction and Educational Role. Springer, Dordrecht, Netherlands. DOI:https://doi. org/10.1007/978-94-017-9496-1_2

[14] TurtleBot. 2021. TurtleBot3 Waffle Pi. Retrieved September 21, 2021 from https://www.turtlebot. $\mathrm{com} /$

[15] Adelheid Voskuhl. 2007. Producing objects, producing texts: accounts of android automata in late eighteenth-century Europe. Studies in History and Philosophy of Science Part A 38, 2 (June 2007), 422-444. DOI:https://doi.org/10.1016/j. shpsa.2007.03.004

[16] Wikipedia. 2021. Diorama. Retrieved from https:// en.wikipedia.org/w/index.php?title=Diorama \&oldid $=1040503092$ 